\title{
TITLE:
}

\section{A Coordinate-Based Proof of the Scallop Theorem}

$\operatorname{AUTHOR}(S)$ :

Ishimoto, Kenta; Yamada, Michio

CITATION:

Ishimoto, Kenta ...[et al]. A Coordinate-Based Proof of the Scallop

Theorem. SIAM Journal on Applied Mathematics 2012, 72(5): 1686-1694

ISSUE DATE:

2012-10

URL:

http://hdl.handle.net/2433/161797

RIGHT:

(c) 2012 Society for Industrial and Applied Mathematics 


\title{
A COORDINATE-BASED PROOF OF THE SCALLOP THEOREM*
}

\author{
KENTA ISHIMOTO $^{\dagger}$ AND MICHIO YAMADA ${ }^{\dagger}$
}

\begin{abstract}
We reconsider fluid dynamics for a self-propulsive swimmer in Stokes flow. With an exact definition of deformation of a swimmer, a coordinate-based proof is first given to Purcell's scallop theorem including the body rotation.
\end{abstract}

Key words. Stokes flow, scallop theorem, microorganism

AMS subject classifications. 76Z10, 74F10, 76D07

DOI. $10.1137 / 110853297$

1. Introduction. Fluid dynamics of locomotion of microorganisms such as bacteria and plankton has been studied for more than half a century [12], [17] and is still a hot topic in physics, mathematics, and biology [9]. A milestone discussion of the locomotion of such microswimmers is the well-known Purcell's scallop theorem [14], which asserts that a microorganism with a reciprocal stroke in Stokes fluid cannot travel in one movement but must exert thrust against the flow via a nonreciprocal stroke. The proof of the theorem given in Purcell's famous lecture [14] was only schematic. Although the theorem was repeatedly discussed by many researchers [1], [2], [4], [7], [15], the definition of the deformation of the swimmer appears not to have been given much attention.

Shapere and Wilczek [15] first established a theoretical formalism for a swimmer in viscous fluid in terms of gauge structure and gave a proof for the scallop theorem. In the case of zero inertia, the motion of the swimmer is determined from the position and the orientation of the swimmer at the same instant, irrespective of the history of the swimmer's motion. Therefore, if a swimmer's surface deformation is the same as every other swimmer's, the motions of all swimmers are related only by translation and rotation, which are often called the gauge structure of their motion. In [15], the displacement and rotation are given by a line integral of a matrix, which is called gauge potential, over configurations of the swimmer in a shape space. Then they gave a proof to the scallop theorem, employing a finite curve without self-intersection in the shape space as the integral path. In their framework, however, no operational formulae for the locomotion is provided, since the definition of the surface deformation of the swimmer is not explicitly given, and therefore it is not clearly distinguished from the net movement, i.e., the translation and the rotation of the swimmer.

Yariv [18] introduced two frames of reference for decomposing the velocity into surface deformation and the net movement. The first is an inertial reference frame and the second is a "body-fixed" frame. The origin of the latter was taken at its geometric center, while he commented that it is possible to take the origin at an arbitrary position. Then he derived an operational formula which gives its position and orientation in terms of the surface deformation labeled by Lagrangian coordinates. However, he only discussed the motion without rotation and he did not prove the scallop theorem completely. As pointed out by Yariv [18] himself, the rotation and the

\footnotetext{
${ }^{*}$ Received by the editors October 28, 2011; accepted for publication (in revised form) July 30, 2012; published electronically October 25, 2012.

http://www.siam.org/journals/siap/72-5/85329.html

${ }^{\dagger}$ Research Institute for Mathematical Sciences, Kyoto University, Kitashirakawaoiwake-cho, Sakyo-ku, Kyoto 606-8502, Japan (ishimoto@kurims.kyoto-u.ac.jp, yamada@kurims.kyoto-u.ac.jp).
}

1686 
surface deformation of the swimmer are not uniquely identified, although he suggested another proof in which the swimmer is isolated from the surrounding fluid. The arbitrariness of the decomposition causes a difficulty in his proof when the swimmer rotates.

In this paper, we will give a definition of the surface deformation of the swimmer, introducing a virtual swimmer which undergoes the same surface deformation as the real swimmer but has no ambient fluid. Our definition identifies the surface deformation uniquely, and then we prove the scallop theorem including a rotational motion of the swimmer. We call the coordinates attached to the virtual swimmer the vacuum coordinates, and those attached to the real swimmer the body coordinates. The transformation from the former to the latter defines the displacement and the rotation (i.e., the gauge) of the swimmer. It should be remarked that deformation of the virtual swimmer conserves the momentum and the angular momentum. By use of the vacuum and the body coordinates, we will consider the case of mass-zero limit to prove the scallop theorem.

A remark should be made on the nonzero mass case, which has also been a subject of research recently. On the status of the scallop theorem, Childress and Dudley [3] stated

As far as we know there has been no rigorous proof of this theorem based upon the mechanics of a Navier-Stokes fluid and free-swimming body

and discussed the case of nonzero inertia of both a body and fluid, and argued the possibility of a sudden breakdown of the theorem at a nonzero critical Reynolds number. The breakdown of the theorem may also arise from unreciprocal deformation and from fluid properties such as rheology [10] and inertia [3], [8]. The scallop theorem and its breakdown have been reviewed recently by Lauga [11].

The breakdown due to a finite mass of the swimmer, i.e., a finite Stokes number, was first discussed by Gonzalez-Rodriguez and Lauga [5]. They provided general differential equations that govern locomotion of such a dense swimmer, and gave some examples of swimmers which negate the theorem due to the body inertia. However, in their research, the definition of the deformation of the swimmer was not uniquely defined.

This paper consists of four sections. Section 1 is the introduction, and in section 2 we define a uniquely identified deformation of a swimmer. Then we discuss a theoretical framework for a swimmer immersed in Stokes fluid, and give an equation that governs the locomotion of the swimmer, by use of the reciprocal theorem following Stone and Samuel [16] and Yariv [18]. In section 3 we restate the scallop theorem and give a complete proof of it. A summary and discussion are given in section 4 .

2. Formulation. In this section we set up formulae for velocity and angular velocity of a self-propulsive swimmer in a fluid. To discuss the motion of the swimmer, we first define a virtual swimmer, which deforms its body in exactly the same way as the real swimmer, except that the virtual swimmer has no surrounding fluid and thus experiences no external forces. The virtual swimmer therefore conserves the total momentum and the total angular momentum, both of which we assume to be zero. We further assume that at the initial time the virtual swimmer exactly coincides with the real swimmer, and therefore both their centers of mass are located at the same position, and their orientations are the same. We attach inertial coordinates, which we call the vacuum coordinates, to this virtual swimmer with its origin located at the center of mass. The virtual swimmer has exactly the same shape as the real swimmer 


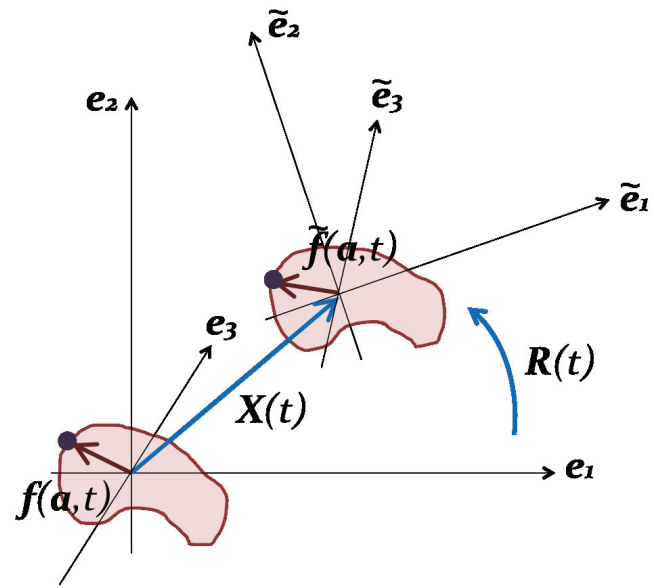

FIG. 2.1. The vacuum coordinates $\left\{\boldsymbol{e}_{i}\right\}$ and the body coordinates $\left\{\tilde{\boldsymbol{e}}_{i}\right\}$, the latter of which is given by the rotational transformation from the former coordinates: $\tilde{\boldsymbol{e}}_{i}(t)=\mathbf{R}(t) \cdot \boldsymbol{e}_{i}$, where $\mathbf{R}$ is the rotational matrix.

at the same time, and thus the latter is obtained by an affine transformation from the former. We also define the body coordinates attached to the real swimmer as the coordinates obtained by the same affine transformation from the vacuum coordinates. Thus the origin of the body coordinates is located at the center of mass of the real swimmer. We denote the orthonormal basis of the vacuum coordinates by $\boldsymbol{e}_{i}(i=$ $1,2,3$ ), which is independent of time (Figure 2.1). The motion of the virtual swimmer is described in Lagrangian coordinates where a position of a Lagrangian particle of the virtual swimmer, $\boldsymbol{f}(\boldsymbol{a}, t)=\sum_{i} f_{i}(\boldsymbol{a}, t) \boldsymbol{e}_{i}$, is regarded as a function of the Lagrangian coordinates $\boldsymbol{a}=\left(a_{1}, a_{2}, a_{3}\right)$ and time $t$ with $\boldsymbol{f}(\boldsymbol{a}, 0)=\boldsymbol{a}$.

We now define the surface deformation velocity $\boldsymbol{u}^{\prime}$ of the virtual swimmer as $\boldsymbol{u}^{\prime}=\sum_{i} \frac{\partial f_{i}}{\partial t}(\boldsymbol{a}, t) \boldsymbol{e}_{i}$, where $\boldsymbol{a}$ is assumed to be on the surface of the swimmer. The real swimmer, however, not only deforms but also translates and rotates under the action of the external force from the surrounding fluid. The translation velocity $\boldsymbol{U}$ is defined as $\boldsymbol{U}=d \boldsymbol{X} / d t$ where $\boldsymbol{X}(t)$ is the center of mass of the real swimmer. The position of the Lagrangian particle of the real swimmer $\tilde{\boldsymbol{f}}(\boldsymbol{a}, t)$ with respect to the center of mass of the real swimmer is then obtained as $\tilde{\boldsymbol{f}}(\boldsymbol{a}, t)=\mathbf{R}(t) \cdot \boldsymbol{f}(\boldsymbol{a}, t)$ or $\tilde{f}_{i}=R_{i j} f_{j}$, where $\mathbf{R}(t)$ is a rotation matrix in $S O(3)$ and $\mathbf{R}(0)=\mathbf{1}$, because the real and virtual swimmers have exactly the same shape. The Einstein convention for repeated indices is employed.

The rotational angular velocity vector $\boldsymbol{\Omega}(t)$ is defined together with two skew symmetric matrices $\mathbf{A}(t), \mathbf{B}(t)$ as

$$
\begin{aligned}
\frac{d \mathbf{R}(t)}{d t} & =\mathbf{B}(t) \cdot \mathbf{R}(t)=\mathbf{R}(t) \cdot \mathbf{A}(t)=\mathbf{\Omega} \times \mathbf{R}(t), \\
\Omega_{i}(t) & =-\epsilon_{i j k} B_{j k}(t) .
\end{aligned}
$$

The total surface velocity $\boldsymbol{u}$ of the real swimmer is

$$
\boldsymbol{u}=\boldsymbol{U}+\frac{\partial \tilde{\boldsymbol{f}}}{\partial t}=\boldsymbol{U}+\boldsymbol{\Omega} \times \tilde{\boldsymbol{f}}+\tilde{\boldsymbol{u}}^{\prime}
$$

where we have used (2.1). The last term $\tilde{\boldsymbol{u}}^{\prime}$ means the surface deformation velocity of the real swimmer defined as $\tilde{\boldsymbol{u}}^{\prime}=\mathbf{R} \cdot \boldsymbol{u}^{\prime}$. 
The governing equation of the fluid surrounding the real swimmer is the incompressible Navier-Stokes equation. The equation for fluid velocity $\boldsymbol{v}=\boldsymbol{v}(\boldsymbol{x}, t)$ is written in a nondimensional form as follows:

$$
\begin{aligned}
\nabla \cdot \boldsymbol{\Pi} & =R_{\omega} \frac{\partial \boldsymbol{v}}{\partial t}+R e(\boldsymbol{v} \cdot \nabla) \boldsymbol{v} \\
\nabla \cdot \boldsymbol{v} & =0
\end{aligned}
$$

and the stress tensor $\boldsymbol{\Pi}$ is given by

$$
\boldsymbol{\Pi}=-p \mathbf{1}+\left(\nabla \boldsymbol{v}+(\nabla \boldsymbol{v})^{\mathrm{T}}\right) .
$$

The nondimensional parameters of the equation, $R e$ and $R_{\omega}$, are the Reynolds number $R e=\rho V L / \mu$ and the oscillatory Reynolds number $R_{\omega}=\rho L^{2} \omega / \mu$, the latter of which corresponds to the product of the Reynolds number and the Strouhal number $S t=L \omega / V$, where $L, V$, and $\omega$ are, respectively, the characteristic scales of length, velocity, and frequency $(d / d t)$ of the fluid motion, with the density $\rho$ and the viscosity $\mu$ of the fluid being assumed constant in this paper. The boundary condition for $\boldsymbol{v}$ at the body surface of the real swimmer is that $\boldsymbol{v}$ coincides with the total surface velocity $\boldsymbol{u}$ of the real swimmer, while $\boldsymbol{v} \rightarrow 0$ at infinity.

From Newton's equation of motion for the real swimmer, we obtain the following equation in the nondimensional form,

$$
R_{S} \frac{d}{d t}\left(\begin{array}{c}
\boldsymbol{U} \\
\tilde{\mathbf{I}} \cdot \boldsymbol{\Omega}
\end{array}\right)=\left(\begin{array}{c}
\boldsymbol{F} \\
\boldsymbol{T}
\end{array}\right)
$$

where $\tilde{\mathbf{I}}$ is the inertial momentum tensor of the real swimmer. The nondimensional number $R_{S}$, the Stokes number, is represented as $R_{S}=\rho_{M} L^{2} \omega / \mu$, where $\rho_{M}$ is the mean density of the swimmer. The force $\boldsymbol{F}$ and the torque $\boldsymbol{T}$ acting on the real swimmer are also nondimensionalized like $\boldsymbol{F}^{*}=\mu L V \boldsymbol{F}$ using the Stokes law of resistance, where the asterisk denotes the dimensional quantity. This nondimensionalization is effective in a situation without other external forces such as gravity and electromagnetic forces.

Hereafter, we assume that the nondimensional parameters $R e \mathrm{C}, R_{\omega} \mathrm{C} R_{S}$ satisfy the inequality $R e, R_{\omega}, R_{S} \ll 1$. In this section we will consider the motion of the swimmer in the fluid and will derive equations for the velocity $\boldsymbol{U}$ and the angular velocity $\boldsymbol{\Omega}$ using a method similar to that of Stone and Samuel [16] and Yariv [18]. When the fluid obeys the steady Stokes equation, Lorentz' reciprocal theorem [13] gives

$$
\int_{\tilde{S}} d \tilde{S} \boldsymbol{n} \cdot \boldsymbol{\Pi} \cdot \hat{\boldsymbol{u}}=\int_{\tilde{S}} d \tilde{S} \boldsymbol{n} \cdot \hat{\boldsymbol{\Pi}} \cdot \boldsymbol{u},
$$

where $\tilde{S}$ and $d \tilde{S}$ denote the surface and the surface element of the real swimmer, while we describe those of the virtual swimmer by $S$ and $d S$. The "hat" symbol indicates quantities of another solution of the Stokes equations with the same boundary shape $\tilde{S}$, and the vector $\boldsymbol{n}$ denotes the unit normal vector to the surface. For the solution $\hat{\boldsymbol{u}}$ we take a solution satisfying the boundary condition

$$
\hat{\boldsymbol{u}}=\hat{\boldsymbol{U}}+\hat{\boldsymbol{\Omega}} \times \tilde{\boldsymbol{f}}
$$


at the surface $S$ of the swimmer [16]. With linearity of the Stokes equation, the stress tensor is given by

$$
\hat{\boldsymbol{\Pi}}=\tilde{\boldsymbol{\Sigma}}_{T} \cdot \hat{\boldsymbol{U}}+\tilde{\boldsymbol{\Sigma}}_{R} \cdot \hat{\boldsymbol{\Omega}},
$$

where $\tilde{\boldsymbol{\Sigma}}_{T}$ and $\tilde{\boldsymbol{\Sigma}}_{R}$ are third rank tensors [6] which depend on the direction of the body coordinates $\tilde{\boldsymbol{e}}_{i}$. Substituting (2.9) and (2.10) into (2.8), we have

$$
\left(\begin{array}{c}
\boldsymbol{F} \\
\boldsymbol{T}
\end{array}\right) \cdot\left(\begin{array}{l}
\hat{\boldsymbol{U}} \\
\hat{\boldsymbol{\Omega}}
\end{array}\right)=\int_{\tilde{S}} d \tilde{S}\left(\begin{array}{l}
\left(\boldsymbol{n} \cdot \tilde{\boldsymbol{\Sigma}}_{T}\right)^{\mathrm{T}} \cdot \boldsymbol{u} \\
\left(\boldsymbol{n} \cdot \tilde{\boldsymbol{\Sigma}}_{R}\right)^{\mathrm{T}} \cdot \boldsymbol{u}
\end{array}\right) \cdot\left(\begin{array}{c}
\hat{\boldsymbol{U}} \\
\hat{\boldsymbol{\Omega}}
\end{array}\right),
$$

where the superscript $\mathrm{T}$ denotes the transpose of the tensor. Here $\boldsymbol{n} \cdot \tilde{\boldsymbol{\Sigma}}_{T}$ and $\boldsymbol{n} \cdot \tilde{\boldsymbol{\Sigma}}_{R}$ denote the second rank tensors $n_{i} \tilde{\Sigma}_{T i j k}$ and $n_{i} \tilde{\Sigma}_{R i j k}$, respectively. Using the $6 \times 6$ symmetric resistive tensor ${ }^{1}$

$$
\tilde{\mathcal{K}}=\left(\begin{array}{ll}
\tilde{\mathbf{K}}_{T}, & \tilde{\mathbf{K}}_{C}^{T} \\
\tilde{\mathbf{K}}_{C}, & \tilde{\mathbf{K}}_{R}
\end{array}\right)=\int_{\tilde{S}} d \tilde{S}\left(\begin{array}{cc}
\boldsymbol{n} \cdot \tilde{\boldsymbol{\Sigma}}_{T}, & \boldsymbol{n} \cdot \tilde{\boldsymbol{\Sigma}}_{R} \\
\tilde{\boldsymbol{f}} \times\left(\boldsymbol{n} \cdot \tilde{\boldsymbol{\Sigma}}_{T}\right), & \tilde{\boldsymbol{f}} \times\left(\boldsymbol{n} \cdot \tilde{\boldsymbol{\Sigma}}_{R}\right)
\end{array}\right)
$$

and arbitrariness of $\hat{\boldsymbol{U}}$ and $\hat{\boldsymbol{\Omega}}$, we obtain

$$
\left(\begin{array}{l}
\boldsymbol{F} \\
\boldsymbol{T}
\end{array}\right)=\tilde{\mathcal{K}} \cdot\left(\begin{array}{l}
\boldsymbol{U} \\
\boldsymbol{\Omega}
\end{array}\right)+\int_{\tilde{S}} d \tilde{S}\left(\begin{array}{l}
\left(\boldsymbol{n} \cdot \tilde{\boldsymbol{\Sigma}}_{T}\right)^{\mathrm{T}} \cdot \tilde{\boldsymbol{u}}^{\prime} \\
\left(\boldsymbol{n} \cdot \tilde{\boldsymbol{\Sigma}}_{R}\right)^{\mathrm{T}} \cdot \tilde{\boldsymbol{u}}^{\prime}
\end{array}\right)
$$

With negligible small Stokes number in Newton's equation of motion (2.7), we obtain the desired equation:

$$
\begin{aligned}
0 & =\left(\begin{array}{l}
\boldsymbol{F}_{T R} \\
\boldsymbol{T}_{T R}
\end{array}\right)+\left(\begin{array}{l}
\boldsymbol{F}_{D} \\
\boldsymbol{T}_{D}
\end{array}\right), \\
\left(\begin{array}{l}
\boldsymbol{F}_{T R} \\
\boldsymbol{T}_{T R}
\end{array}\right) & =\tilde{\mathcal{K}} \cdot\left(\begin{array}{l}
\boldsymbol{\Omega} \\
\boldsymbol{\Omega}
\end{array}\right), \\
\left(\begin{array}{l}
\boldsymbol{F}_{D} \\
\boldsymbol{T}_{D}
\end{array}\right) & =\int_{\tilde{S}} d \tilde{S}\left(\begin{array}{l}
\left(\boldsymbol{n} \cdot \tilde{\boldsymbol{\Sigma}}_{T}\right)^{\mathrm{T}} \cdot \tilde{\boldsymbol{u}}^{\prime} \\
\left(\boldsymbol{n} \cdot \tilde{\boldsymbol{\Sigma}}_{R}\right)^{\mathrm{T}} \cdot \tilde{\boldsymbol{u}}^{\prime}
\end{array}\right) .
\end{aligned}
$$

The force $\boldsymbol{F}_{T R}$ and the torque $\boldsymbol{T}_{T R}$ are the external force and the torque from the fluid, arising from the translation and rotation of the real swimmer, while the force $\boldsymbol{F}_{D}$ and the torque $\boldsymbol{T}_{D}$ arise from the surface deformation of the swimmer. We remark that the right-hand side of (2.14) represents the total force and torque exerted on the real swimmer, and therefore when we take into account the gravity and the buoyancy effects, we need only add the gravity and the buoyancy forces to $\boldsymbol{F}$ and their torques to $\boldsymbol{T}$, respectively.

We should note that both the third rank tensors, $\tilde{\boldsymbol{\Sigma}}_{T}$ and $\tilde{\boldsymbol{\Sigma}}_{R}$, and the resistive tensor $\tilde{\mathcal{K}}$ of the real swimmer depend only on the surface shape of the real swimmer $\tilde{\boldsymbol{f}}(t)$.

As we are considering the scallop theorem, which is concerned with the motion of the swimmer due to its surface deformation defined by using the virtual swimmer,

\footnotetext{
${ }^{1}$ The relation of off-diagonal components of $\mathcal{K}$ is as below using Lorentz' reciprocal theorem. If we take two solutions with the boundary condition $\boldsymbol{u}=\boldsymbol{U}$ and $\hat{\boldsymbol{u}}=\boldsymbol{\Omega} \times \boldsymbol{f}$, respectively, at an arbitrary surface $S$, we obtain $\boldsymbol{\Pi}=\boldsymbol{\Sigma}_{T} \cdot \boldsymbol{U}$ and $\hat{\boldsymbol{\Pi}}=\boldsymbol{\Sigma}_{R} \cdot \boldsymbol{\Omega}$. Substitution of these into equation (2.8) gives $\int_{S} d S \boldsymbol{n} \cdot\left(\boldsymbol{\Sigma}_{T} \cdot \boldsymbol{U}\right) \cdot(\boldsymbol{\Omega} \times \boldsymbol{f})=\int_{S} d S \boldsymbol{n} \cdot\left(\boldsymbol{\Sigma}_{\boldsymbol{R}} \cdot \boldsymbol{\Omega}\right) \cdot \boldsymbol{U}$. After some manipulations, we get $\int_{S} d S \boldsymbol{\Omega} \cdot \boldsymbol{f} \times\left(\boldsymbol{n} \cdot \boldsymbol{\Sigma}_{T}\right) \cdot \boldsymbol{U}=\int_{S} d S \boldsymbol{\Omega} \cdot\left(\boldsymbol{n} \cdot \boldsymbol{\Sigma}_{R}\right)^{\mathrm{T}} \cdot \boldsymbol{U}$, which implies that the transpose of an off-diagonal component is another off-diagonal component.
} 
it is convenient to describe the problem in terms of quantities of the virtual swimmer in the vacuum coordinates. We then have the equations

$$
0=\mathcal{K} \cdot \mathcal{R}^{-1} \cdot\left(\begin{array}{l}
\boldsymbol{U} \\
\boldsymbol{\Omega}
\end{array}\right)+\int_{S} d S\left(\begin{array}{l}
\left(\boldsymbol{n} \cdot \boldsymbol{\Sigma}_{T}\right)^{\mathrm{T}} \cdot \boldsymbol{u}^{\prime} \\
\left(\boldsymbol{n} \cdot \boldsymbol{\Sigma}_{R}\right)^{\mathrm{T}} \cdot \boldsymbol{u}^{\prime}
\end{array}\right),
$$

where the $6 \times 6$ matrix $\mathcal{R}$ is defined as

$$
\mathcal{R}=\left(\begin{array}{ll}
\mathbf{R} & 0 \\
0 & \mathbf{R}
\end{array}\right) .
$$

When the deformation velocity of the virtual swimmer is given, this equation together with (2.1) determines the rotation matrix $\mathbf{R}(t)$ and the translational velocity $\boldsymbol{U}(t)$.

3. Proof of the scallop theorem. In this section we give a proof of the scallop theorem using (2.1) and (2.17).

We consider the case where the shape of the virtual swimmer deforms in a reciprocal manner; i.e., the shape once deformed retraces back to the initial shape. The mathematical definition of the reciprocal motion is that for the surface deformation of the virtual swimmer, which starts at $t=0$ and ends at $t=T$, there exists a continuous function $g(t)$ such that $\boldsymbol{f}(t)=\mathbf{Q}(t) \cdot \boldsymbol{f}(g(t))$ and $g(0)=g(T)=0$, where $\mathbf{Q}(t) \in S O(3)$ is a three-dimensional rotation matrix, allowing for the possibility that the swimmer takes different directions at time $t$ and $g(t)$. We assume that $g(t)$ is smooth except at a finite number of points, and then the intervals of integration over the time $t$ in the following should be divided into those in which $g(t)$ remains smooth. In this case, we can prove that $\mathbf{Q}(t)=\mathbf{1}$ as below.

The total angular momentum of the virtual swimmer always vanishes, and therefore,

$$
\mathbf{0}=\int \rho_{m} \boldsymbol{f}(\boldsymbol{a}, t) \times \frac{\partial \boldsymbol{f}}{\partial t}(\boldsymbol{a}, t) d \boldsymbol{a}=\int \rho_{m} \mathbf{Q} \cdot \boldsymbol{f}(\boldsymbol{a}, g(t)) \times \frac{\partial \mathbf{Q} \cdot \boldsymbol{f}(\boldsymbol{a}, g(t))}{\partial t} d \boldsymbol{a} .
$$

Be sure that the order of the substitution and the differentiation are indicated by the positions of the parentheses and the differentiation, respectively; for example, $\frac{\partial \boldsymbol{f}(g(t))}{\partial t}$ is equal to $\left.\frac{\partial \boldsymbol{f}\left(t^{\prime}\right)}{\partial t^{\prime}}\right|_{t^{\prime}=g(t)} \frac{d g(t)}{d t}$, while $\frac{\partial \boldsymbol{f}}{\partial t}(g(t))$ is equal to $\left.\frac{\partial \boldsymbol{f}\left(t^{\prime}\right)}{\partial t^{\prime}}\right|_{t=g(t)}$. Noting that

$$
\frac{\partial \mathbf{Q} \cdot \boldsymbol{f}(\boldsymbol{a}, g(t))}{\partial t}=\boldsymbol{\Omega}^{Q} \times \boldsymbol{f}(\boldsymbol{a}, t)+\frac{d g}{d t} \mathbf{Q} \cdot \frac{\partial \boldsymbol{f}}{\partial t}(\boldsymbol{a}, g(t)),
$$

where the angular velocity vector $\Omega^{Q}$ is defined as

$$
\frac{d \mathbf{Q}}{d t}=\mathbf{\Omega}^{Q} \times \mathbf{Q}, \quad \Omega_{i}^{Q}=-\epsilon_{i j k}\left(\frac{d \mathbf{Q}}{d t} \cdot \mathbf{Q}^{-1}\right)_{j k},
$$

and $\rho_{m}=\rho_{m}(\boldsymbol{a})$ is the density of the swimmer, we find the contribution from the second term of (3.2) to (3.1) vanishes as

$$
\begin{aligned}
\int \rho_{m} \mathbf{Q}(t) \cdot \boldsymbol{f}(\boldsymbol{a}, g(t)) \times \frac{d g}{d t} \mathbf{Q} \cdot \frac{\partial \boldsymbol{f}}{\partial t}(\boldsymbol{a}, g(t)) d \boldsymbol{a} \\
=\frac{d g}{d t} \mathbf{Q}(t) \cdot \int \rho_{m} \boldsymbol{f}(\boldsymbol{a}, g(t)) \times \frac{\partial \boldsymbol{f}}{\partial t}(\boldsymbol{a}, g(t)) d \boldsymbol{a}=0
\end{aligned}
$$

Copyright $@$ by SIAM. Unauthorized reproduction of this article is prohibited. 
due to the vanishing initial angular momentum conserved. Then (3.2) gives

$$
\int \rho_{m} \boldsymbol{f}(\boldsymbol{a}, t) \times\left(\boldsymbol{\Omega}^{Q}(t) \times \boldsymbol{f}(\boldsymbol{a}, t)\right) d \boldsymbol{a}=\mathbf{I}(t) \cdot \boldsymbol{\Omega}^{Q}(t)=0,
$$

where $\mathbf{I}$ is the inertial momentum tensor of the virtual swimmer. For a general threedimensional swimmer, $\mathbf{I}$ is not degenerated and we have $\boldsymbol{\Omega}^{Q}(t)=0$. Then (3.3) with $\mathbf{Q}(0)=1$ gives $\mathbf{Q}(t)=\mathbf{1}$.

The scallop theorem asserts that the position and the direction of the real swimmer at the final time $t=T$ coincide with the initial position and direction if the motion of the swimmer is reciprocal, the surrounding fluid obeys the steady Stokes equation, and the Stokes number of the swimmer vanishes. In this case, (2.17) is reduced to

$$
\left(\begin{array}{l}
\boldsymbol{U} \\
\boldsymbol{\Omega}
\end{array}\right)=-\mathcal{R} \cdot \mathcal{M} \cdot \int_{S} d S\left(\begin{array}{l}
\left(\boldsymbol{n} \cdot \boldsymbol{\Sigma}_{T}\right)^{\mathrm{T}} \cdot \boldsymbol{u}^{\prime} \\
\left(\boldsymbol{n} \cdot \boldsymbol{\Sigma}_{R}\right)^{\mathrm{T}} \cdot \boldsymbol{u}^{\prime}
\end{array}\right),
$$

where the tensor $\mathcal{M}$, the mobility tensor, is the inverse of the resistive tensor $\mathcal{K}$.

Denoting $t^{\prime}=g(t)$ for short, (3.6) is reduced to

$$
\begin{aligned}
\left(\begin{array}{l}
\boldsymbol{U}(t) \\
\boldsymbol{\Omega}(t)
\end{array}\right) & =-\mathcal{R}(t) \cdot \mathcal{M}(t) \cdot\left(\begin{array}{l}
\int_{S(t)} d S\left(\boldsymbol{n} \cdot \boldsymbol{\Sigma}_{T}\right)^{\mathrm{T}}(t) \cdot \boldsymbol{u}^{\prime}(t) \\
\int_{S(t)} d S\left(\boldsymbol{n} \cdot \boldsymbol{\Sigma}_{R}\right)^{\mathrm{T}}(t) \cdot \boldsymbol{u}^{\prime}(t)
\end{array}\right) \\
& =-\mathcal{R}(t) \cdot \mathcal{M}\left(t^{\prime}\right) \cdot\left(\begin{array}{l}
\int_{S\left(t^{\prime}\right)} d S\left(\boldsymbol{n} \cdot \boldsymbol{\Sigma}_{T}\right)^{\mathrm{T}}\left(t^{\prime}\right) \cdot \boldsymbol{u}^{\prime}\left(t^{\prime}\right) \\
\int_{S\left(t^{\prime}\right)} d S\left(\boldsymbol{n} \cdot \boldsymbol{\Sigma}_{R}\right)^{\mathrm{T}}\left(t^{\prime}\right) \cdot \boldsymbol{u}^{\prime}\left(t^{\prime}\right)
\end{array}\right) \frac{d t^{\prime}}{d t} \\
& =\mathcal{R}(t) \cdot \mathcal{R}^{-1}\left(t^{\prime}\right) \cdot\left(\begin{array}{l}
\boldsymbol{U}\left(t^{\prime}\right) \\
\boldsymbol{\Omega}\left(t^{\prime}\right)
\end{array}\right) \frac{d t^{\prime}}{d t}
\end{aligned}
$$

where we have used the fact that $\mathcal{M}(t)=\mathcal{M}\left(t^{\prime}\right), S(t)=S\left(t^{\prime}\right), \boldsymbol{\Sigma}_{T}(t)=\boldsymbol{\Sigma}_{T}\left(t^{\prime}\right)$, and $\boldsymbol{\Sigma}_{R}(t)=\boldsymbol{\Sigma}_{R}\left(t^{\prime}\right)$, which holds because $\boldsymbol{f}(\boldsymbol{a}, t)=\boldsymbol{f}\left(\boldsymbol{a}, t^{\prime}\right)$. Especially from this equation, we obtain

$$
\mathbf{R}^{-1}(t) \cdot \boldsymbol{\Omega}(t)=\mathbf{R}^{-1}\left(t^{\prime}\right) \cdot \boldsymbol{\Omega}\left(t^{\prime}\right) \frac{d t^{\prime}}{d t}
$$

Using (2.1) and (2.2) we obtain

$$
A_{i j}(t)=R_{i l}^{-1} B_{l k} R_{k j}=-\epsilon_{l k p} R_{l i} R_{k j} \Omega_{p}
$$

and from the definition of the determinant of the matrix $\mathbf{R}$ we have

$$
\epsilon_{l k p} R_{l i} R_{k j} R_{p q}=|\mathbf{R}| \epsilon_{i j q}
$$

which leads to

$$
\epsilon_{l k r} R_{l i} R_{k j}=\epsilon_{i j q} R_{r q}=\epsilon_{i j q}\left(\mathbf{R}^{-1}\right)_{q r}
$$

by the use of $|\mathbf{R}|=1$ and the multiplication of $R_{r q}$ to (3.12). Substituting (3.13) into (3.11), we have

$$
A_{i j}(t)=-\epsilon_{i j k}\left(\mathbf{R}^{-1}(t) \cdot \boldsymbol{\Omega}(t)\right)_{k},
$$

Copyright (C) by SIAM. Unauthorized reproduction of this article is prohibited. 
which together with (3.10) gives

$$
\mathbf{A}(t)=\mathbf{A}\left(t^{\prime}\right) \frac{d t^{\prime}}{d t}
$$

This relation reflects the gauge structure of the system. The matrix $\mathbf{A}$ corresponds to a part of a vector potential $A^{\text {rot }}$, which is described in equation (2.5) of [15]. The rotation matrix $\mathbf{R}(t)$ satisfies

$$
\frac{d \mathbf{R}(t)}{d t}=\mathbf{R}(t) \cdot \mathbf{A}(t),
$$

and $\mathbf{R}(g(t))$ is also the solution of (3.16), because

$$
\frac{d \mathbf{R}(g(t))}{d t}=\frac{d g(t)}{d t} \frac{d \mathbf{R}}{d t}(g(t))=\frac{d g(t)}{d t} \mathbf{R}(g(t)) \cdot \mathbf{A}(g(t))=\mathbf{R}(g(t)) \cdot \mathbf{A}(t)
$$

and $\mathbf{R}(g(0))=\mathbf{R}(0)=\mathbf{1}$. Therefore $\mathbf{R}(g(t))=\mathbf{R}(t)$, and thus ${ }^{2} \mathbf{R}(g(T))=\mathbf{R}(0)=\mathbf{1}$.

Using this relation in (3.9), we have $\boldsymbol{U}(t)=\boldsymbol{U}\left(t^{\prime}\right) d t^{\prime} / d t$ which implies

$$
\frac{d \boldsymbol{X}(g)}{d t}=\frac{d g(t)}{d t} \frac{d \boldsymbol{X}}{d t}(g(t))=\frac{d g(t)}{d t} \boldsymbol{U}(g(t))=\boldsymbol{U}(t) .
$$

Together with $\boldsymbol{X}(g(0))=\boldsymbol{X}(0)$, this means that $\boldsymbol{X}(g(t))=\boldsymbol{X}(t)$ and thus $\boldsymbol{X}(T)=$ $\boldsymbol{X}(g(T))=\boldsymbol{X}(0)=\mathbf{0}$, which completes the proof of the scallop theorem.

4. Summary and discussion. We have established a framework for discussing a motion of a swimmer immersed in Stokes fluid, and have given a complete proof for the scallop theorem.

In order to define the deformation velocity, we introduced the virtual swimmer which has the same shape as the real swimmer but has no ambient fluid. We then attached the vacuum coordinates to the virtual swimmer and the body coordinates to the real swimmer. The position and the orientation of the real swimmer are obtained by an affine transformation from the virtual swimmer. We derived the formulae which provide the velocity and the angular velocity of the swimmer when the surface deformation of the virtual swimmer is given in the case where Reynolds number $R e$, oscillatory Reynolds number $R_{\omega}$, and Stokes number $R_{S}$ vanish. Using these formulae, we have proved the scallop theorem at a vanishing mass of the swimmer or, equivalently, a zero Stokes number.

Our theoretical framework, which gives a uniquely determined surface deformation, is applicable also to the swimmer with nonzero mass. Letting the Stokes number in Newton's equation (2.7) be a nonzero constant, the equation for the swimmer (2.17) becomes

$$
R_{S} \frac{d}{d t}\left(\begin{array}{c}
\boldsymbol{U} \\
\mathbf{R} \cdot \mathbf{I} \cdot \mathbf{R}^{-1} \cdot \boldsymbol{\Omega}
\end{array}\right)=\mathcal{R} \cdot \mathcal{K} \cdot \mathcal{R}^{-1} \cdot\left(\begin{array}{l}
\boldsymbol{U} \\
\boldsymbol{\Omega}
\end{array}\right)+\mathcal{R} \cdot \int_{S} d S\left(\begin{array}{l}
\left(\boldsymbol{n} \cdot \boldsymbol{\Sigma}_{T}\right)^{\mathrm{T}} \cdot \boldsymbol{u}^{\prime} \\
\left(\boldsymbol{n} \cdot \boldsymbol{\Sigma}_{R}\right)^{\mathrm{T}} \cdot \boldsymbol{u}^{\prime}
\end{array}\right)
$$

With this equation, a perturbational approach is possible for the breakdown of the scallop theorem due to the nonzero mass. This analysis shows that the order of the displacement in a period of the reciprocal deformation is related to a symmetry of the surface deformation of the swimmer, which will be reported elsewhere.

\footnotetext{
${ }^{2}$ This can be proved also by noticing that $\mathbf{R}(T)=\mathbf{R}(0) \overline{\mathrm{T}} e^{\int_{0}^{T} \mathbf{A}(t) d t}=\mathbf{R}(0) \overline{\mathrm{T}} e^{\int_{0}^{0} \mathbf{A}\left(t^{\prime}\right) d t^{\prime}}=\mathbf{1}$, where $\int_{0}^{0}$ symbolizes the round integration from $t^{\prime}=0$ to $t^{\prime}=0$, and $\bar{T}$ is the anti-time-ordering operator.
} 
Acknowledgments. We would like to thank Prof. Takehiro for providing us with carefully considered feedback and valuable comments. Special thanks are also due to Mr. Inubushi, Mr. Kimura, Ms. Obuse, and Mr. Sasaki, who gave us invaluable comments and warm encouragement.

\section{REFERENCES}

[1] T. Chambrion and A. Munnier, Generalized Scallop Ttheorem for Linear Swimmers, preprint, 2010, arXiv 1008.1098v1[math-ph].

[2] S. Childress, Mechanics of Swimming and Flying, Cambridge University Press, New York, 1981.

[3] S. Childress AND R. Dudley, Transition from ciliary to flapping mode in a swimming mollusc: Flapping flight as a bifurcation in Re $e_{\omega}$, J. Fluid Mech., 498 (2004), pp. 257-288.

[4] A. DeSimone, F. Alouges, and A. Lefebvre, Biological Fluid Dynamics: Swimming at Low Reynolds Numbers, Preprint 21, SISSA, Trieste, Italy, 2008.

[5] D. Gonzalez-Rodriguez and E. Lauga, Reciprocal locomotion of dense swimmers in Stokes flow, J. Phys. Condens. Matter, 21 (2009), 204103.

[6] J. Happel And H. BRenner, Low Reynolds Number Hydrodynamics with Special Applications to Particulate Media, Martinus Nijhoff Publishers, The Hague, The Netherlands, 1983.

[7] L. Koller, K. Ehlers, and R. Montgomery, Problems and progress in microswimming, J. Nonlinear Sci., 6 (1996), pp. 507-541.

[8] E. LAUGA, Continuous breakdown of Purcell's scallop theorem with inertia, Phys. Fluid, 19 (2007), 061703

[9] E. Lauga, The hydrodynamics of swimming microorganisms, Rep. Prog. Phys., 72 (2009), 096601.

[10] E. Lauga, Life at high Deborah number, Euro. Phys. Lett., 86 (2009), 64001.

[11] E. LAUGA, Life around the scallop theorem, Soft Matter, 7 (2011), pp. 3060-3065.

[12] J. Lighthill, Flagellar hydrodynamics, SIAM Rev., 18 (1976), pp. 161-230.

[13] H. A. Lorentz, Ein allgemeiner Satz, die Bewegung einer reibenden Flüssigkeit betreffend, nebst einigen Anwendungen desselben, Abhandl. Theoret. Phys., 1 (1906), pp. 23-42.

[14] E. M. Purcell, Life at low Reynolds number, Amer. J. Phys., 45 (1977), pp. 3-11.

[15] A. Shapere AND F. WilczeK, Geometry of self-propulsion at low Reynolds number, J. Fluid Mech., 198 (1989), pp. 557-585.

[16] H. A. Stone And A. D. T. Samuel, Propulsion of microorganisms by surface distortions, Phys. Rev. Lett., 77 (1996), pp. 4102-4104.

[17] S. G. TAYLOR, Analysis of the swimming of microscopic organisms, Proc. Roy. Soc. London Ser. A, 209 (1951), pp. 447-461.

[18] E. YARIV, Self-propulsion in a viscous fluid: Arbitrary surface deformations, J. Fluid Mech., 550 (2006), pp. 139-148.

Copyright (C) by SIAM. Unauthorized reproduction of this article is prohibited. 